\title{
X-ray waveguide nanostructures: Design, fabrication, and characterization
}

\author{
A. Jarre, J. Seeger, C. Ollinger, and C. Fuhse \\ Institute for X-ray physics, ${ }^{\mathrm{a}}$ Georg-August University Göttingen, D37077 Göttingen, Germany \\ C. David \\ Laboratory for Micro- and Nanotechnology, Paul Scherrer Institut, 5232 Villigen, Switzerland \\ T. Salditt ${ }^{\mathrm{b})}$ \\ Institute for X-ray Physics, Georg-August University Göttingen, D-37077 Göttingen, Germany
}

(Received 17 July 2006; accepted 15 December 2006; published online 5 March 2007)

\begin{abstract}
Two dimensionally confining $\mathrm{x}$-ray channel waveguide structures are fabricated and used for the delivery of nanoscopic x-ray beams. The waveguides can be combined with a high gain Kirkpatrick-Baez-prefocusing mirror system yielding hard $\mathrm{x}$-ray beams with a cross section down to $25 \mathrm{~nm}$ (full width at half maximum). The incoming synchrotron $\mathrm{x}$-ray beam is coupled in from the front side of the waveguide. Here we address the general design of the x-ray optical devices and their fabrication by e-beam lithography methods. () 2007 American Institute of Physics. [DOI: $10.1063 / 1.2435943$ ]
\end{abstract}

\section{INTRODUCTION}

Focusing hard $\mathrm{x}$-rays down to spot sizes in the range of $10-100 \mathrm{~nm}$ has become an active field of research, but still presents considerable experimental challenges. Such x-ray nanobeams enable a variety of imaging techniques, such as scanning fluorescence, scanning transmission microscopy in phase and/or absorption contrast, as well as in-line holography. A variety of approaches are currently pursued to achieve hard x-ray nanobeams such as Fresnel zone plates (FZPs), ${ }^{1}$ compound refractive lenses (CRLs), ${ }^{2,3}$ bent mirrors in Kirkpatrick-Baez (KB) geometry, ${ }^{4,5}$ as well as combinations of these techniques, e.g., Bragg Fresnel lenses on curved multilayer mirrors. ${ }^{6}$ We have recently demonstrated the feasibility of a two dimensional beam-confinement based on an $\mathrm{x}$-ray channel waveguide (WG). ${ }^{7}$ In this setup an incoming focused beam is directed on the front side of a buried lithographic channel structure-in contrast to the previously used resonant beam coupling geometry, ${ }^{8-10}$ where the incoming beam is resonantly coupled in from the side of the channel through a thinned cladding. Front-coupled waveguides do not provide a significant flux density enhancement (gain) by themselves, but rather act as ultranarrow slits which further reduce the size of a (pre-)focused beam to the desired small values. At the same time it blocks all unwanted radiation by absorption in the cladding. The mode structure leads to radiation fields which are precisely defined and spatially coherent, at least in the limit of a single excited mode. ${ }^{11}$ Thus, the WG acts as a spatial and coherence filter defining the beam properties. The prefocused beam is coupled directly into the front side of the waveguide and can be harvested at its exit with its known computable profile, see Fig. 1. Bergemann et $a l{ }^{12}$ have shown that the minimum cross section $W_{C}$ of the exiting beam is limited by the material combination of the device. For Si-polymethymetacrylate (PMMA) waveguides $W_{C}$ is in the range of $20 \mathrm{~nm}$.

\footnotetext{
a)URL: http://www.roentgen.physik.uni-goettingen.de/

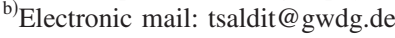

In the present paper we give details of the experiment reported earlier in a Letter, ${ }^{7}$ with a special emphasis on the fabrication of the WG nanostructures. WGs for front coupling need to block all unwanted radiation originating from those parts of the focused beam which over illuminate the WG entrance-the geometric acceptance of the guiding channel is some tens of nanometers only. These requirements lead to structures of high aspect ratio (length of the device over its width or height). The fabrication of such structures by e-beam lithography (EBL) is described in Sec. III, following a general discussion on waveguide design. An experimental characterization of several fabricated devices is presented in Sec. IV.

\section{WAVEGUIDE DESIGN}

$\mathrm{X}$-ray waveguides consist of at least two materials with refractive indices $n_{j}=1-\delta_{j}-i \beta_{j}$, where $\delta_{j}$ is the refractive index decrement $\left(\delta \sim 10^{-5}-10^{-8}\right.$ for hard x rays) and $\beta_{j}$ is the imaginary part of the refractive index responsible for the absorption in material $j$. The guiding material of an $\mathrm{x}$-ray waveguide is the material with smaller $\delta$, enabling internal

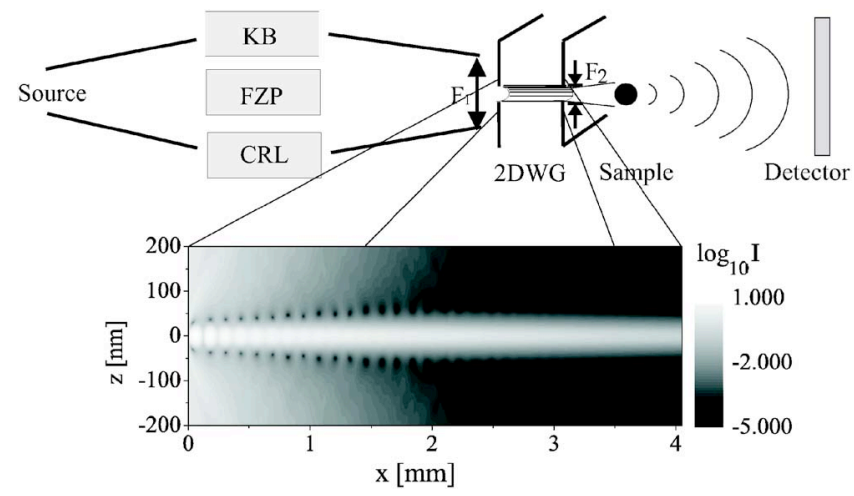

FIG. 1. Schematic of a front-coupling x-ray waveguide setup. The waveguide is positioned in the focus of a high gain optical system, e.g., KB, FZP, or CRL. A numerical simulation of the field propagation in the waveguide is shown below in logarithmic gray scales. 


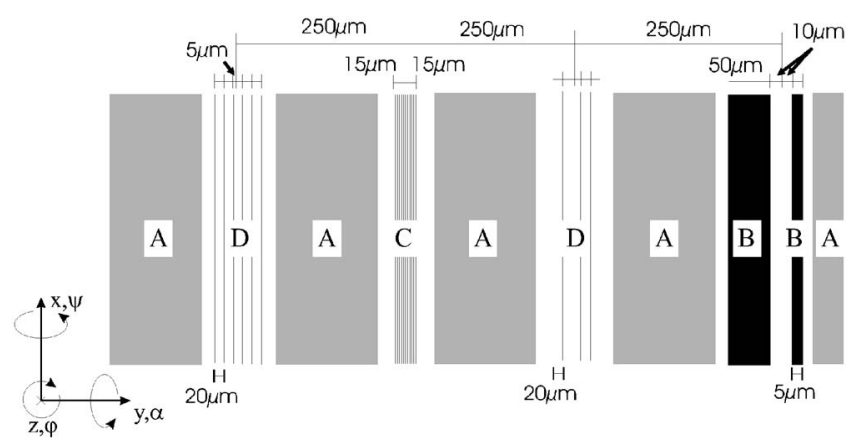

FIG. 2. Design of an e-beam lithography pattern comprising of (A) broad one dimensionally confining waveguides (1DWGs), (B) smaller 1DWGs, (C) waveguide gratings (WGGs), and (D) two dimensionally confining waveguides (2DWGs), all on the same silicon substrate.

total reflection. Such structures allow for the propagation of a defined number $N$ of modes, where $N$ depends on the refractive indices of the materials and on the geometric parameters. ${ }^{13}$ The difference $\Delta \delta$ of the refractive index decrements delimits the minimum size of the guided beam. The modes can be excited, e.g., by shining an x-ray beam of wave number $|\mathbf{k}|=2 \pi / \lambda$ directly onto the waveguide entrance (front coupling). In this setup, the wave vector $\mathbf{k}$ must lie inside a narrow cone around the propagation direction of the guide. The opening angle of the cone corresponds to the angular acceptance of the waveguide and is in the range of the critical angle, i.e., a few milliradians. Therefore, efficient prefocusing optics providing small spot sizes may well be used to increase the flux density in front of the guide, if the convergence of the impinging beam does not exceed the waveguide angular acceptance. The alignment of a single nanometer sized two dimensionally confining WG (2DWG) gets more and more difficult as the size of the impinging beam decreases. Therefore, additional "prealignment" structures must be fabricated next to the 2DWGs. For waveguides produced by EBL, one dimensionally confining planar waveguides and linear gratings made up of several 2DWGs (Ref. 14) are fabricated on the same wafer to facilitate the alignment in the beam.

EBL is used to fabricate the desired patterns enabling a precise control of the relative positions of the respective guides, which is important for the alignment. In Fig. 2(a) possible design of such a multiwaveguide device is shown which can be prepared on standard $\mathrm{Si}$ wafers: The gray shaded areas (A) represent large one dimensionally confining WGs (1DWGs) which allow for the alignment of two incidence angles $\alpha_{i}$ and $\Psi_{i}$. While a single 1DWG is used to align $\alpha_{i}$, a second 1DWG, preferably located far away from the first one, helps to determine the misalignment of $\Psi$. Then, the front side of the waveguide device can be scanned vertically through the focal spot of the focusing optics. The alignment is then refined on the smaller 1DWGs (B) and the waveguide grating (WGG), which is particularly useful to find the zero in $\varphi$. Note that both elements still exhibit a significantly higher throughput than a single 2DWG (D), which cannot be aligned directly. Thus, the small 1DWGs and the grating are used consecutively to align the incidence angle $\varphi$ before a 2DWG is finally positioned in the focal

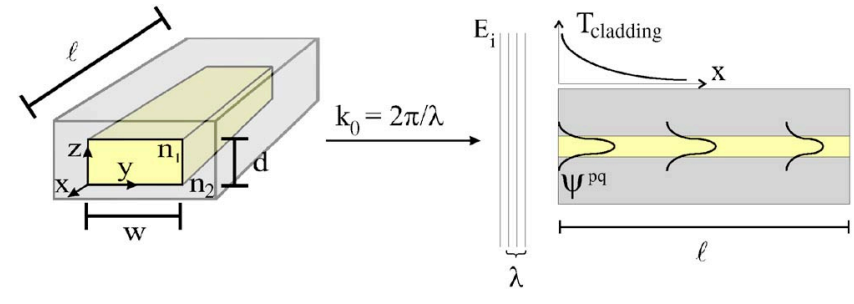

FIG. 3. Schematic of the channel waveguide and coupling geometry.

spot. Following this procedure, a $2 \mathrm{DWG}$ alignment can be accomplished within a few hours. Rapid selection between different 2DWGs oriented perfectly parallel on the chip is then easily possible. Note that the procedure requires a two dimensional camera to monitor the transmitted beam in the two directions simultaneously. A video readout of an optical camera directed on a scintillation foil turned out to be very useful.

Let us briefly consider beam propagation in $\mathrm{x}$-ray waveguides. For rectangular core geometries as shown in Fig. 3, i.e., for refractive index profiles given by

$$
n(y, z)= \begin{cases}n_{1}, & \text { for } 0 \leqslant z \leqslant d \text { and } 0 \leqslant y \leqslant w \\ n_{2}, & \text { else }\end{cases}
$$

the Helmholtz equation can be written for radiation polarized along the $y$ axis as

$$
\frac{\partial^{2} E_{y}(y, z)}{\partial y^{2}}+\frac{\partial^{2} E_{y}(y, z)}{\partial z^{2}}+\left[n(y, z)^{2} k^{2}-\beta^{2}\right] E_{y}(y, z)=0 .
$$

Note that the $y$ axis of the waveguides denotes the horizonal direction, along which undulator radiation is polarized. More generally, the Helmholtz equation should be written for all polarization states. ${ }^{13}$ Note, however, that for $\mathrm{x}$ rays with $n$ $\simeq 1$ all solutions degenerate to the same field distribution which can easily be obtained from the scalar wave theory. However, Eq. (2) cannot be solved analytically. Yet, neglecting the corner regions (to the top left of the core, the top right, bottom left, and bottom right), analytical approximations can be made with high accuracy. In this approximation, these solutions essentially factorize into the independent solutions of two orthogonal one dimensionally confining waveguides. With decreasing core size, however, the contributions of the corner regions will increase. Therefore a numerical approach is used to describe the field distribution in the device. If the wave vector of the incident beam is restricted to incidence angles which are less than the critical angle $\alpha_{c}$ away from the optical $(x)$ axis $\left(\alpha_{i}<\alpha_{c}\right)$, the Helmholtz equation can be solved in the approximation of the parabolic wave equation,

$$
2 i k_{0} \frac{\partial u}{\partial x}+\frac{\partial^{2} u}{\partial y^{2}}+\frac{\partial^{2} u}{\partial z^{2}}+k_{0}^{2}\left(n^{2}-1\right) u=0,
$$

where $u$ is defined by $\Psi(x, y, z)=u(x, y, z) e^{-i k_{0} x}$. Equation (3) can be solved numerically by finite-difference calculations ${ }^{15}$ calculating the field distribution step by step over the entire length of the guide. 


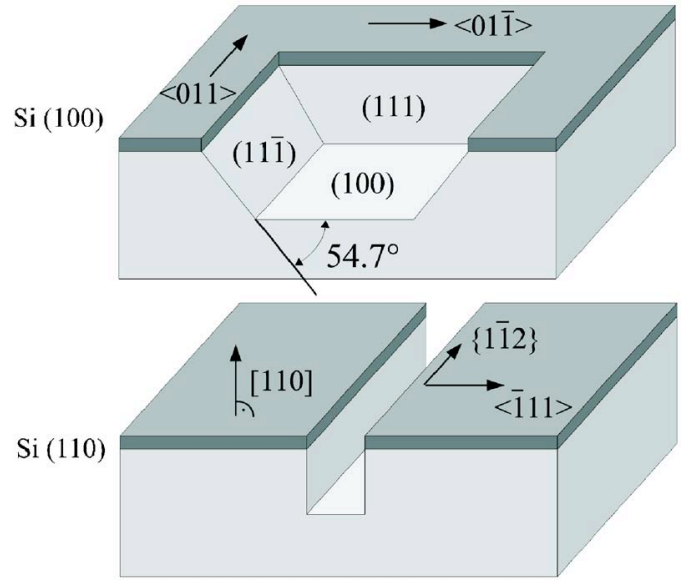

FIG. 4. Since KOH etches Si anisotropically, the etching results in V-shaped or U-shaped grooves, depending on the crystal orientation of the Si wafer.

\section{WAVEGUIDE FABRICATION}

Using e-beam lithography for waveguide fabrication, we may chose between direct and indirect fabrication methods. The direct fabrication is comparatively straight forward: The desired waveguide pattern is written into an e-beam positive resist such as PMMA (exposed areas are washed away during the development). The pattern is then developed and the resulting structure is covered by an evaporated $\mathrm{Si}$ layer of some hundred nanometer thickness. After breaking the wafer to the desired length - determined by the required absorption of the primary beam $(\mathrm{PB})$ as a function of overillumination and photon energy - the waveguide is ready for use. However, with this method we are restricted to e-beam resists as guiding material which slightly decreases the maximum refractive index decrement difference $\Delta \delta$ between the core and the cladding when compared to air or vacuum guiding in the same cladding. If desired, this limitation can be overcome by an indirect fabrication method: The waveguide pattern is again defined by EBL. This pattern is then transferred into the Si wafer. Therefore a layer system is needed which allows for subsequent transfer of the pattern from one layer to the next. After pattern transfer, the effect of anisotropic etching of $\mathrm{Si}$ by $\mathrm{KOH}$ can be used to define the waveguide geometry (see Fig. 4).

In a $\mathrm{Si}$ crystal the etching speed of $\mathrm{KOH}$ in direction perpendicular to the $\{111\}$ plane is reduced by one to two orders of magnitude when compared to the etching speed in all other directions. ${ }^{16,17}$ Therefore, $\mathrm{KOH}$ etching will result in U- or V-shaped grooves when applied to (110)- or (100)oriented wafers, respectively. In the case of V-shaped grooves, the opening angle of the "V" is fixed to $70.5^{\circ}$, which limits the depth of the groove to $1 / \sqrt{2}$ times its width. In contrast, for the U-groove fabrication the ratio between the depth of a groove and its width is ultimately limited by the ratio of the etching speeds of $\mathrm{KOH}$ in the $\langle 111\rangle$ and the $\langle 110\rangle$ directions. The layer system follows the concept of using the $n$th layer (counted from the top) as an etch mask for the $n+1$ st layer, see Fig. 5 .

Since $\mathrm{SiO}_{2}$ is not affected by $\mathrm{KOH}$, it can act as an etch mask for the final step, transferring the pattern into the wafer. $\mathrm{SiO}_{2}$ in turn can be etched by HF which does not affect $\mathrm{Si}$. Si

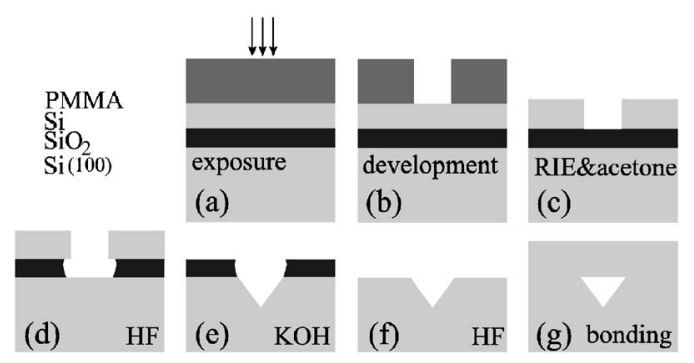

FIG. 5. (a) Layer system used for V-groove fabrication. PMMA acts as positive resist in EBL. After (b) the development of the pattern, it is (c) transferred into the evaporated $\mathrm{Si}$ by reactive ion etching in $\mathrm{CBrF}_{3}$ atmosphere. Residual PMMA is washed away with acetone. (d) The oxide layer is etched isotropically with $\mathrm{HF}$, the remaining $\mathrm{Si}$ above the oxide is taken away (e) in a subsequent $\mathrm{KOH}$ bath which at the same time etches the V-groove into the (100)-oriented $\mathrm{Si}$ wafer. (f) The oxide is removed by another HF bath. (g) The groove is covered by bonding a second Si wafer on top of the structured one.

thus acts as a mask for $\mathrm{SiO}_{2}$. This $\mathrm{Si}$ layer can be etched anisotropically by reactive ion etching (RIE) using $\mathrm{CBrF}_{3}$. A sufficiently thick PMMA layer on top of the Si can act as a mask in the RIE and can therefore be used to write the desired pattern with an electron beam. After development, the pattern is transferred through $\mathrm{Si}$ and $\mathrm{SiO}_{2}$ into the wafer. Note, however, that the $\mathrm{SiO}_{2}$ etching with $\mathrm{HF}$ is isotropic and causes broadening of the lines due to underetching of the above Si layer (Fig. 5(d)). Therefore, it is especially important to minimize the etching time in order to reduce this effect.

\section{A. Materials and methods}

\section{Direct structuring}

Si wafers were bought from Silchem, Germany. Before further processing they were cleaned following the method described in Ref. 18, known as RCA1 and RCA2. The native oxide was removed in a $20 \mathrm{~s}$ concentrated HF bath at room temperature. The PMMA solution for spincoating was prepared by solving 1-5 g PMMA (Elvacite 2041, Lehmann \& Voss \& Co, molecular weight: $450000 \mathrm{~g} / \mathrm{mol}$ ) in $100 \mathrm{ml}$ 2-methoxy-ethyl-acetate providing a large range of possible

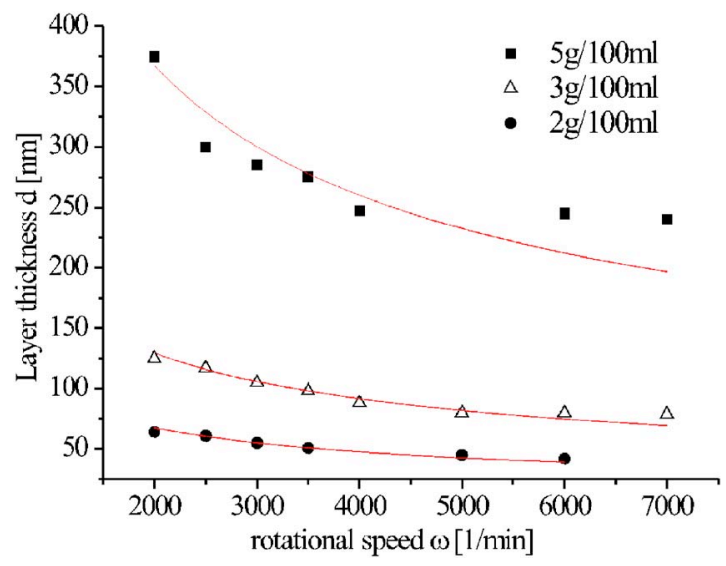

FIG. 6. Sketch of the dependence of the final polymer layer thickness $d$ on the polymer concentration $c$ (PMMA, amount in $\mathrm{g}$ ) in its solvent $(100 \mathrm{ml}$ 2-methoxy-ethyl-acetate (MEA)) and on the rotational speed $\omega$ during the spincoating process. Lines are best fits to the data. 

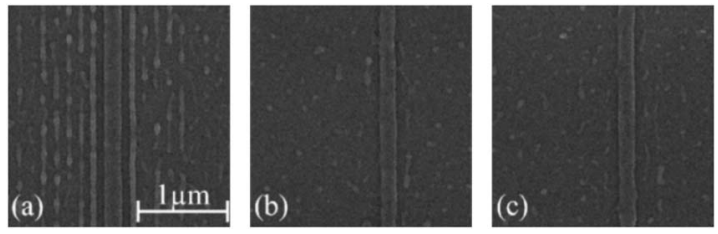

FIG. 7. SEM micrographs taken during the preparation of single PMMA stripes for direct 2DWG fabrication on a Si (100) wafer with $77 \mathrm{~nm}$ PMMA after e-beam lithography and development.

spincoating layer thicknesses (see Fig. 6) following the relation $d(\mathrm{~nm})=686 c^{2} / \omega^{1 / 2},{ }^{19}$ where $\omega$ is the rotational speed in $\min ^{-1}$ and $c$ is the polymer concentration in grams per $100 \mathrm{ml}$ solvent. The exposed film was developed in a 4:1 mixture of ethyleneglycolmonoethylether and ethyleneglycolmonobutylether.

1DWG fabrication is especially convenient in positive resists since it is achieved by simply leaving broad areas unexposed. The fabrication of a $2 \mathrm{DWG}$ in turn requires the exposure of large areas around a single unexposed stripe of PMMA. Area exposure is achieved by writing parallel lines at small distance $d_{\text {area }}$, while the polymer stripe is generated by a one-time change from the small line distance to a larger one $\left(d_{\text {stripe }}\right)$, i.e., a single jump during the area exposure. To determine the exposure parameters, in particular, an adequate line dose (LD) and a line distance for a given jump width, e.g., $d_{\text {stripe }}=150 \mathrm{~nm}$, the effect of different LDs is analyzed experimentally. Figure 7 shows the scanning electron microscopy (SEM) micrographs of three structures after the line fabrication processes, i.e., after development, illustrating the influence of LD and line distance $d_{\text {area }}$ on the resulting stripe width $w_{\text {stripe }}$. The patterns were generated at the following parameters: (a) $d_{\text {area }}=70 \mathrm{~nm}$ and $\mathrm{LD}=0.175$ $\mathrm{nC} / \mathrm{cm} \Rightarrow w_{\text {stripe }}=95 \mathrm{~nm}, \quad$ (b) $\quad d_{\text {area }}=70 \mathrm{~nm}$ and LD $=0.200 \mathrm{~nm} \Rightarrow w_{\text {stripe }}=88 \mathrm{~nm}$, and (c) $d_{\text {area }}=60 \mathrm{~nm}$ and LD $=0.175 \mathrm{nC} / \mathrm{cm} \Rightarrow w_{\text {stripe }}=79 \mathrm{~nm}$.

The preparation of a WGG follows the same principle, but the area exposure reduces to writing not more than two or three lines next to each other before generating a stripe by leaving a wider distance between the exposed lines. The gratings shown in Fig. 8 were generated by writing $n$ lines at a distance $d_{\text {area }}=70 \mathrm{~nm}$ with a LD and jump distance $d_{\text {stripe }}$ $=300 \mathrm{~nm}-n d_{\text {area }}$ (or equivalently with a periodicity $p$ $=300 \mathrm{~nm}$ ), leaving a grating made of single stripes of width $w_{\text {stripe }}$ (a) $n=2, \mathrm{LD}=0.400 \mathrm{nC} / \mathrm{cm}$, and $w_{\text {stripe }}=135 \mathrm{~nm}$, (b) $n=3, \mathrm{LD}=0.300 \mathrm{nC} / \mathrm{cm}$, and $w_{\text {stripe }}=64 \mathrm{~nm}$, and (c) $n=3$, $\mathrm{LD}=0.350 \mathrm{nC} / \mathrm{cm}$, and $w_{\text {stripe }}=$ undetermined.

After the development of the pattern a $\mathrm{Si}$ cap layer of several hundred nanometer thickness is evaporated (Univex 450 , Leybold) to bury the structure with the same material as

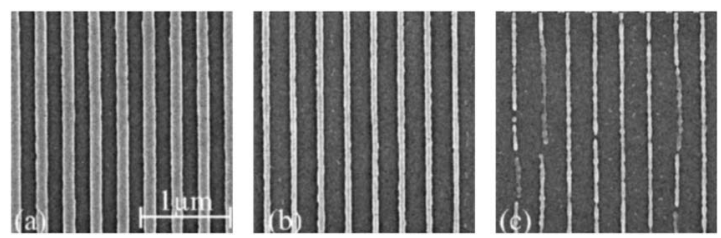

FIG. 8. SEM micrographs of a Si wafer with $77 \mathrm{~nm}$ PMMA spincoated on top after e-beam lithography of a grating and development.

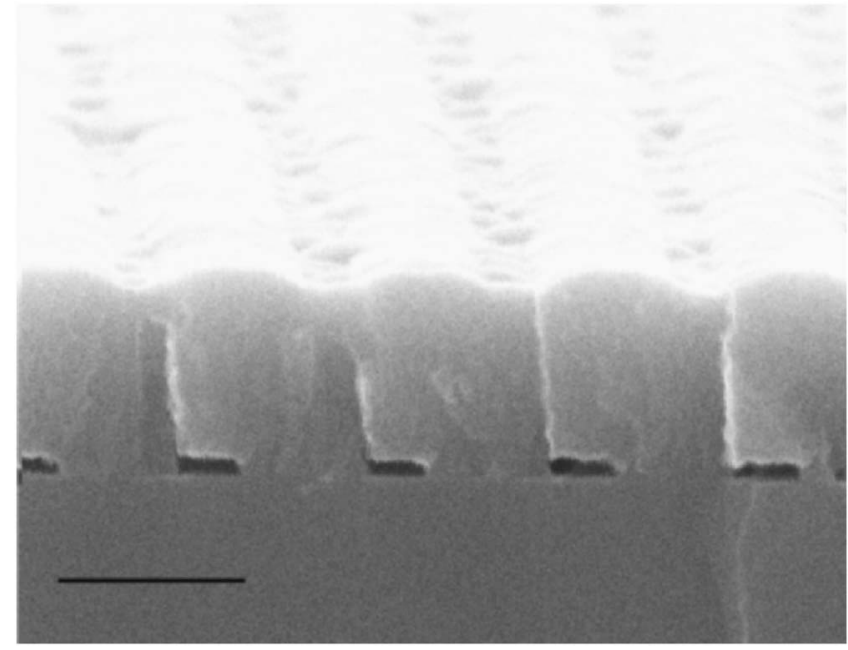

FIG. 9. Micrograph of the front side of a polymer core waveguide grating in $\mathrm{Si}$ after direct fabrication. The scale bar is $300 \mathrm{~nm}$.

the cladding below. Thus, a symmetric profile of the refractive index around the guiding PMMA core is achieved. The device is then ready for use and can be cut to its desired length. The front side of a Si/PMMA WGG is shown in Fig. 9 as observed by SEM.

\section{Indirect structuring}

Si wafers, both (100) and (110) oriented, with an oxide layer of, e.g., $18 \mathrm{~nm}$ thickness, were bought from Silchem. It is advantageous to keep the oxide layer thin to prevent significant broadening of the desired grooves due to the isotropic $\mathrm{HF}$ etching. At the same time it has to be thick enough to serve as an etch mask for the $\mathrm{KOH}$ etch of the underlying silicon. A layer thickness of $18 \mathrm{~nm}$ which is significantly larger than native oxide layers $(2 \mathrm{~nm})$ turns out to be a suitable value. The evaporated $\mathrm{Si}$ layer thickness $d_{\mathrm{Si}}$ on top of the oxide layer was chosen to be $d_{\mathrm{Si}} \sim 50 \mathrm{~nm}$. PMMA can endure RIE with $\mathrm{CBrF}_{3}$ to some extent. However, due to partial damage, its thickness $d_{\text {PMMA }}$ must be adapted to the evaporated Si layer thickness. For the described layer system it is chosen to be $d_{\mathrm{PMMA}} \simeq 150 \mathrm{~nm}$. In Fig. 10, a series of SEM micrographs is shown of two V-groove gratings after different steps of the fabrication process [corresponding to Figs. 5(c), 5(d), and 5(f)]: The top row shows a grating with
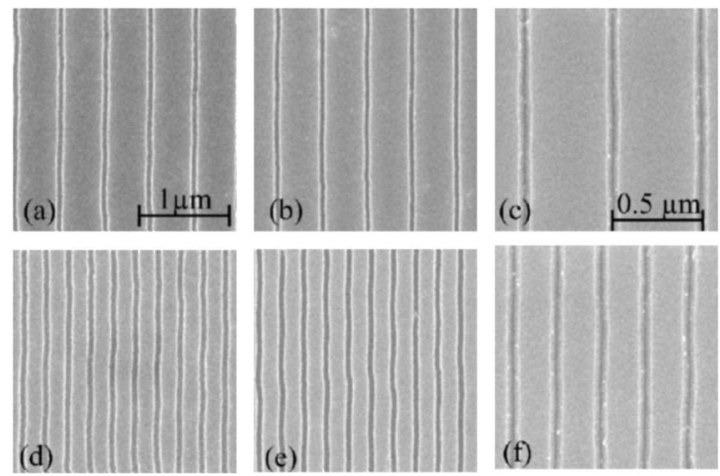

FIG. 10. SEM images of two different gratings (top: periodicity $p$ $=500 \mathrm{~nm}$; bottom: $p=250 \mathrm{~nm}$ ) during V-groove fabrication process. 

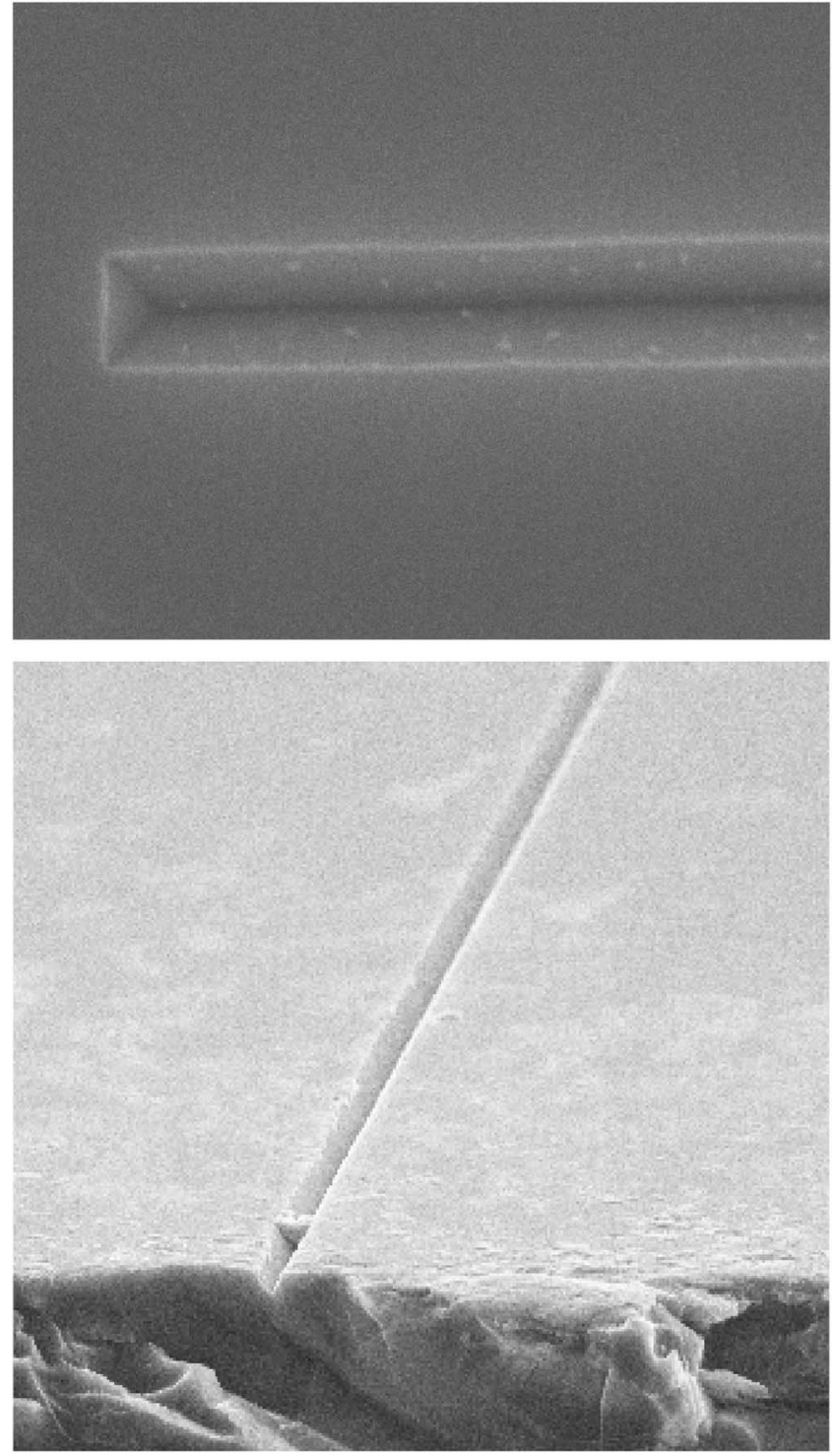

FIG. 11. V-groove as seen by SEM, both in top view and at an angle of $45^{\circ}$.

a periodicity of $500 \mathrm{~nm}$; the periodicity in the bottom row grating is $250 \mathrm{~nm}$. The structures were originally made of a layer system of $150 \mathrm{~nm}$ PMMA on $50 \mathrm{~nm}$ evaporated $\mathrm{Si}$ on $18 \mathrm{~nm} \mathrm{SiO}_{2}$ on a (100)-oriented $\mathrm{Si}$ wafer $\left(10 \times 10 \mathrm{~mm}^{2}\right)$. (a) and (d) show the micrographs taken after lithography, development, and reactive ion etching with $\mathrm{CBrF}_{3}$ [10 cubic centimeter per minute at STP (SCCM), 16 mTorr, $0.2 \mathrm{kV}$ self-bias, and $150 \mathrm{~s}$ ] and subsequent removal of residual PMMA in an ultrasonic acetone bath $(60 \mathrm{~s})$. The width $w_{j}$ of the structure in image $j$ is $w_{a}=60 \mathrm{~nm}$ and $w_{d}=63 \mathrm{~nm}$; (b) and (e) show the same devices after further etching the $\mathrm{SiO}_{2}$ in $1 \mathrm{vol} \% \mathrm{HF}$ at $20^{\circ} \mathrm{C}$ for $135 \mathrm{~s}$ and a $300 \mathrm{~s}$ methanol bath (for cleaning), $w_{b}=w_{e}=63 \mathrm{~nm}$; (c) and (f) (new scale bar) were recorded after etching the $\mathrm{Si}-(100)$ wafer anisotropically in $10 \mathrm{wt} \% \mathrm{KOH}$ for $900 \mathrm{~s}$ at $20{ }^{\circ} \mathrm{C}$ and another bath in $1 \mathrm{vol} \% \mathrm{HF}$ to remove the now dispensable $\mathrm{SiO}_{2}$. $w_{c}=74 \mathrm{~nm}$ and $w_{f}=76 \mathrm{~nm}$.

Figure 11 shows SEM micrographs of V-grooves after completing the fabrication process both in top view and at an angle of $45^{\circ}$. With the same fabrication method but using

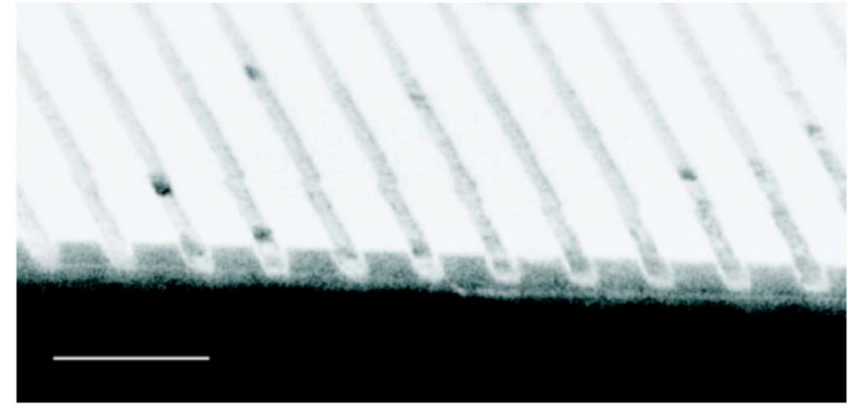

FIG. 12. U-groove grating (scale bar=500 nm) etched into a (110)-oriented Si wafer observed by SEM after removal of the oxide layer. After bonding, gratings can be used to facilitate the alignment of 2DWGs. Gratings also enable the testing of waveguide performance in unfocused beams, i.e., at flux densities which are too low for the examination of a single 2DWG.

(110)-oriented Si wafers, U grooves can be manufactured. This is illustrated in Fig. 12 for a U-groove grating with a periodicity of $250 \mathrm{~nm}$ after RIE $\left(\mathrm{CBrF}_{3}, 10 \mathrm{SCCM}, 0.2 \mathrm{kV}\right.$ self-bias, and $140 \mathrm{~s}), \mathrm{HF}$ ( $1 \mathrm{wt} \%$ and $215 \mathrm{~s}$ ), $\mathrm{KOH}$ U-groove etching (10 wt \%, $15 \mathrm{~min}$ ), and oxide removal in HF ( $1 \mathrm{wt} \%$ and $220 \mathrm{~s}$ ). The layer system consisted of a (110)-oriented $\mathrm{Si}$ wafer, $19 \mathrm{~nm} \mathrm{SiO}_{2}$, evaporated $46 \mathrm{~nm} \mathrm{Si}$ and $150 \mathrm{~nm}$ PMMA on top. The EBL pattern consisted of an array of substructures of two written lines at $\mathrm{LD}=0.18 \mathrm{nC}$ separated by $20 \mathrm{~nm}$. The substructure was repeated at a periodicity of $250 \mathrm{~nm}$.

Once the preparation of U- or V-shaped grooves is completed, the patterned wafer must be capped, preferably with $\mathrm{Si}$, which provides a symmetric refractive index profile. This is best achieved by wafer bonding ${ }^{20}$ since bonding connects the wafer surfaces via intermolecular forces and thus prevents air gaps inbetween the wafer surfaces which would act as 1 DWGs. Note that wafer bonding has recently been demonstrated to achieve buried x-ray waveguide channels. ${ }^{21}$ Figure 13 shows the front side of the V-groove grating in Fig. 10(c) after hydrophobic bonding and annealing.

\section{WAVEGUIDE CHARACTERIZATION}

Waveguides of both types (direct and indirect) have been tested in synchrotron radiation experiments. For selected beamtimes, powerful prefocusing optics and undulator radiation were available, enabling experiments on single channel WGs. The results obtained from PMMA waveguides (direct structuring) in such an experiment have been described previously. ${ }^{7}$ An exiting beam of $25 \times 47 \mathrm{~nm}^{2}$ was obtained with an integral count rate of $4 \times 10^{6}$ counts/s, corresponding to a WG transmission of $\sim 5 \%$. In other experiments,

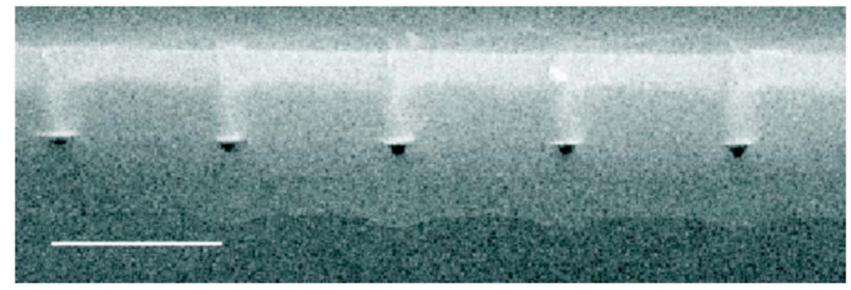

FIG. 13. Bonded V-grooves after $4 \mathrm{~h}$ annealing at $1000{ }^{\circ} \mathrm{C}$. The periodicity of the waveguide grating is $500 \mathrm{~nm}$. 

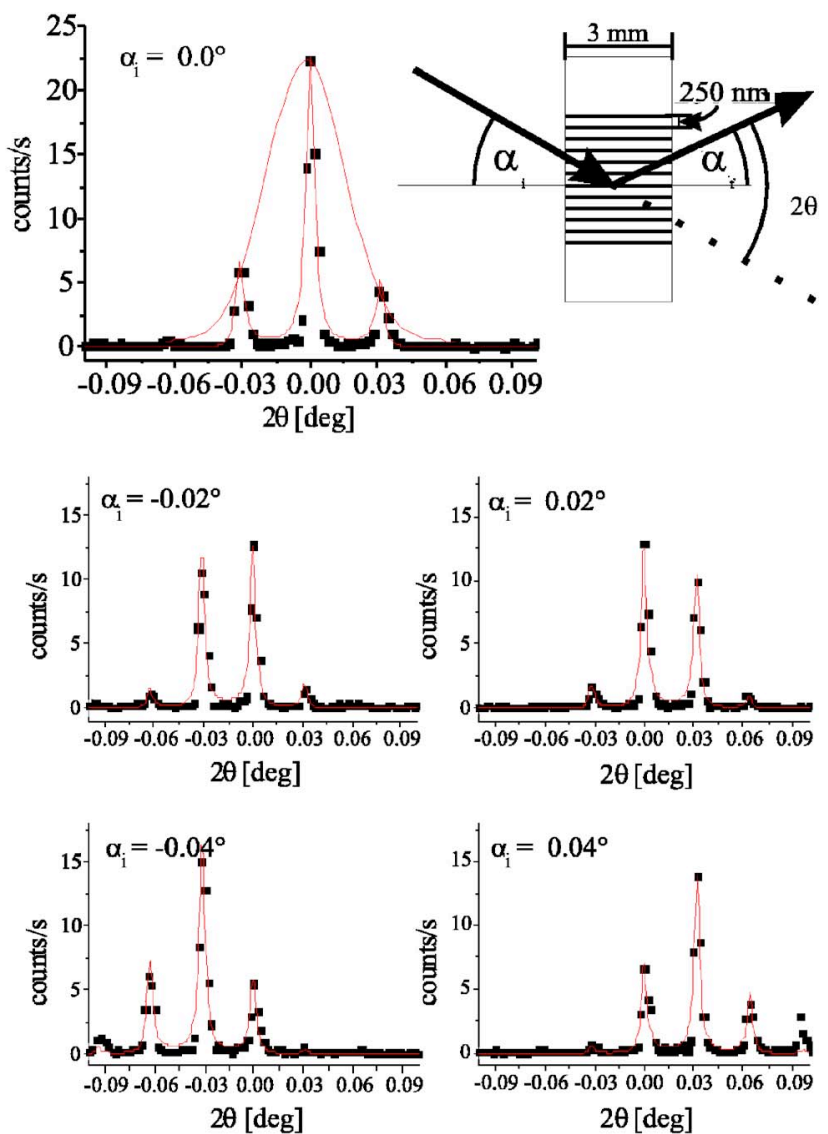

FIG. 14. Measured far-field pattern (symbols) of the U-groove grating in Fig. 12. The inset specifies the angles used to describe the WG far-field measurement. Lines are fits to the data. The envelope in the first graph represents the far field of a single 2DWG of width $w=69.9 \mathrm{~nm}$.

unfocused bending magnet radiation was used. Here we could still characterize the waveguide structures. However, gratings of waveguide channels rather than single channels had to be used, leading to a characteristic far-field interference pattern.

The characterization of waveguide gratings fabricated by U-groove etching and wafer bonding has been performed at the D4.1 bending magnet beamline of the DORIS storage ring at HASYLAB, Hamburg. The photon energy was set to $8.94 \mathrm{keV}$ by a $\mathrm{Ge}$ (111) crystal as measured at the $\mathrm{Yb} \mathrm{L}_{3}$ fluorescence line. Higher harmonics were suppressed by a $\mathrm{Rh}$ mirror tilted to $0.25^{\circ}$. The length $l=3 \mathrm{~mm}$ of the bonded $\mathrm{Si}$ waveguides corresponds to a theoretical transmission of the primary beam through the Si cladding of $T_{\mathrm{Si}}=3 \times 10^{-14}$, 22 which guarantees, that only guided radiation is detected behind the WG.

The far-field interference pattern of the waveguide grating was measured with a scintillation detector (Cyber star, Oxford instruments) placed $679 \mathrm{~mm}$ behind the guides. Figure 14 shows five of the measured far-field patterns for incidence angles $\alpha_{i}$ between $-0.04^{\circ}$ and $0.04^{\circ}$ along with a sketch of the geometry. The grating generates peak intensities in the far field according to the grating relation $d \sin (2 \theta)=n \lambda$, i.e., at $n 0.318^{\circ}$. This structure factor is modulated by the far-field intensity distribution of a single waveguide which represents the envelope function of the overall far field. In other words, only those grating peaks can be detected, which are inside the cone of radiation emitted by each single waveguide. The lines in the graphs represent a fit to the measured data according to (Ref. 14) using a Levenberg-Marquardt algorithm. The fit parameters obtained are in good agreement with the expected values: $d_{\text {fit }}$ $=250.4 \pm 0.2 \mathrm{~nm}$ and $w_{\mathrm{fit}}=69.9 \pm 0.8 \mathrm{~nm}$.

\section{SUMMARY AND CONCLUSION}

Two fabrication methods, direct and indirect structuring, have been described which both provided two dimensionally confining x-ray waveguides. The starting point for both methods is the use of e-beam lithography to achieve lines of high aspect ratio in an adequate resist. Waveguides of both kinds have been tested at synchrotron radiation sources proving the expected optical properties. The main advantage in using the more elaborate indirect structuring method is that air (or vacuum) is used as guiding material, reducing absorption losses and increasing the difference of the refractive indices between the core and the cladding. Both methods can be used to provide sufficiently intense hard x-ray nanobeams at highly brilliant sources, given an effective prefocusing optic.

\section{ACKNOWLEDGMENTS}

We acknowledge the German Federal Ministry of Education and Research (BMBF) for funding through Project No. 05KS4MGA/9. We are grateful for the provision of several consecutive synchrotron beamtimes at HASYLAB/ DESY and ESRF, Grenoble.

${ }^{1}$ B. Nöhammer, C. David, M. Burghammer, and C. Riekel, Appl. Phys. Lett. 86, 163804 (2005).

${ }^{2}$ C. G. Schroer et al., AIP Conf. Proc. 705, 740 (2004).

${ }^{3}$ C. G. Schroer et al., Appl. Phys. Lett. 87, 124103 (2005).

${ }^{4}$ O. Hignette, P. Cloetens, W.-K. Lee, and G. Rostaing, J. Phys. IV 104, 231 (2003).

${ }^{5}$ P. Kirkpatrick and A. V. Baez, J. Opt. Soc. Am. 38, 766 (1948).

${ }^{6}$ M. Yasa, Y. Li, C. Mammen, J. Als-Nielsen, J. Hoszowska, C. Mocuta, and A. Freund, Appl. Phys. Lett. 84, 4744 (2004).

${ }^{7}$ A. Jarre, C. Fuhse, C. Ollinger, R. Toucoulu, and T. Salditt, Phys. Rev. Lett. 94, 074801 (2005).

${ }^{8}$ A. Jarre, T. Salditt, T. Panzner, and U. Pietsch, Appl. Phys. Lett. 85, 161 (2004).

${ }^{9}$ F. Pfeiffer, C. David, M. Burghammer, and T. Salditt, Science 297, 230 (2002).

${ }^{10}$ E. Spiller and A. Segmüller, Appl. Phys. Lett. 24, 60 (1974).

${ }^{11}$ C. Fuhse, A. Jarre, C. Ollinger, J. Seeger, and T. Salditt, Appl. Phys. Lett. 85, 1907 (2004).

${ }^{12}$ C. Bergemann, H. Keymeulen, and J. F. van der Veen, Phys. Rev. Lett. 91, 204801 (2003).

${ }^{13}$ D. Marcuse, Theory of Dielectric Optical Waveguides (Academic, New York, 1974).

${ }^{14}$ C. Ollinger, C. Fuhse, A. Jarre, and T. Salditt, Physica B 357, 53 (2005).

${ }^{15}$ C. Fuhse and T. Salditt, Physica B 357, 57 (2005).

${ }^{16}$ K. Williams and R. Muller, J. Microelectromech. Syst. 4, 256 (1996).

${ }^{17}$ M. Yamamoto, Y. Iriye, K. Asaumi, M. Shikida, Y. Matsushima, T. Yamashiro, and K. Sato, Sens. Actuators, A 64, 87 (1988).

${ }^{18}$ W. Kern, Handbook of Semiconductor Wafer Cleaning (Noyes, Park Ridge, 1993).

${ }^{19}$ C. W. Extrand, Polym. Eng. Sci. 34, 390 (1994).

${ }^{20}$ Q.-Y. Tong and U. Gösele, Semiconductor Wafer Bonding (Wiley, New York, 1998).

${ }^{21}$ M. Poulsen, F. Jensen, O. Bunk, R. Feidenhans'l, and D. W. Breiby, Appl. Phys. Lett. 87, 261904 (2005).

${ }^{22}$ See, e.g., http://www.cxro.lbl.gov. 